\title{
A FINITE DIFFERENCE SOLUTION OF THE REGULARIZED LONG-WAVE EQUATION
}

\author{
S. KUTLUAY AND A. ESEN
}

Received 26 July 2005; Accepted 24 January 2006

A linearized implicit finite difference method to obtain numerical solution of the onedimensional regularized long-wave (RLW) equation is presented. The performance and the accuracy of the method are illustrated by solving three test examples of the problem: a single solitary wave, two positive solitary waves interaction, and an undular bore. The obtained results are presented and compared with earlier work.

Copyright (C) 2006 S. Kutluay and A. Esen. This is an open access article distributed under the Creative Commons Attribution License, which permits unrestricted use, distribution, and reproduction in any medium, provided the original work is properly cited.

\section{Introduction}

In this study, we will consider the one-dimensional RLW equation

$$
\frac{\partial U}{\partial t}+\frac{\partial U}{\partial x}+\varepsilon U \frac{\partial U}{\partial x}-\mu \frac{\partial}{\partial t}\left(\frac{\partial^{2} U}{\partial x^{2}}\right)=0
$$

with the physical boundary conditions $U \rightarrow 0$ as $x \rightarrow \pm \infty$, where $t$ is time, $x$ is the space coordinate, $U(x, t)$ is the wave amplitude, and $\varepsilon$ and $\mu$ are positive parameters. The RLW equation (1.1) was first introduced by Peregrine [1] to describe the development of an undular bore. This equation is one of the most important nonlinear wave equations which can be used to model a large number of problems arising in various areas of applied sciences $[2,3]$. The RLW equation has been solved analytically for a restricted set of boundary and initial conditions. Therefore, the numerical solution of the RLW equation has been the subject of many papers. Various numerical techniques particularly including finite difference [4-8], finite element [9-19], and spectral [20-23] methods have been used for the solution of the RLW equation.

In this paper, we have used a linearized implicit finite difference method to investigate the motion of a single solitary wave, development of two positive solitary waves interaction, and an undular bore for the RLW equation (1.1). 
2 Regularized long-wave equation

\section{Method of solution}

For the numerical treatment, the spatial variable $x$ of the problem is restricted over an interval $a \leq x \leq b$. In this study, we consider the RLW equation (1.1) with the homogeneous boundary conditions

$$
U(a, t)=0, \quad t>0, \quad U(b, t)=0, \quad t>0,
$$

and the initial condition

$$
U(x, 0)=f(x), \quad a \leq x \leq b,
$$

where $f(x)$ is a prescribed function.

The solution domain $a \leq x \leq b, t>0$ is divided into subintervals $\Delta x$ in the direction of the spatial variable $x$ and $\Delta t$ in the direction of time $t$ such that $x_{i}=i \Delta x, i=0(1) N$ $(N \Delta x=b-a) ; t_{j}=j \Delta t, j=0(1) J$, and the numerical solution of $U$ at the grid point $(i \Delta x, j \Delta t)$ is denoted by $U_{i, j}$.

In the finite difference method, the dependent variable and its derivatives are approximated by the finite difference approximation. This approximation will lead to either a single explicit equation or a system of difference equations. Applying the classical implicit finite difference method to nonlinear problems normally gives nonlinear system of equations which cannot be solved directly.

Equation (1.1) can be written as

$$
\frac{\partial U}{\partial t}+\frac{\partial U}{\partial x}+\frac{\varepsilon}{2} \frac{\partial U^{2}}{\partial x}-\mu \frac{\partial}{\partial t}\left(\frac{\partial^{2} U}{\partial x^{2}}\right)=0
$$

Using the forward difference approximation for $\partial U / \partial t$, the Crank-Nicolson difference approximation for $\partial U / \partial x$ and $\partial U^{2} / \partial x$, and the central difference approximation for $\partial^{2} U / \partial x^{2}$ at the point $(i, j+1)$,

$$
\begin{gathered}
\frac{\partial U}{\partial t} \cong \frac{U_{i, j+1}-U_{i, j}}{\Delta t}, \\
\frac{\partial U}{\partial x} \cong \frac{1}{2}\left\{\frac{1}{2 \Delta x}\left(U_{i+1, j+1}-U_{i-1, j+1}\right)+\frac{1}{2 \Delta x}\left(U_{i+1, j}-U_{i-1, j}\right)\right\}, \\
\frac{\partial U^{2}}{\partial x} \cong \frac{1}{2}\left\{\frac{1}{2 \Delta x}\left(U_{i+1, j+1}^{2}-U_{i-1, j+1}^{2}\right)+\frac{1}{2 \Delta x}\left(U_{i+1, j}^{2}-U_{i-1, j}^{2}\right)\right\}, \\
\frac{\partial^{2} U}{\partial x^{2}} \cong \frac{1}{(\Delta x)^{2}}\left(U_{i+1, j}-2 U_{i, j}+U_{i-1, j}\right),
\end{gathered}
$$

respectively, (2.3) yields the system of algebraic equations

$$
\begin{aligned}
& \frac{U_{i, j+1}-U_{i, j}}{\Delta t}+\frac{1}{4 \Delta x}\left(U_{i+1, j+1}-U_{i-1, j+1}+U_{i+1, j}-U_{i-1, j}\right) \\
& \quad+\frac{\varepsilon}{8 \Delta x}\left(U_{i+1, j+1}^{2}-U_{i-1, j+1}^{2}+U_{i+1, j}^{2}-U_{i-1, j}^{2}\right) \\
& \quad-\frac{\mu}{\Delta t(\Delta x)^{2}}\left(U_{i+1, j+1}-2 U_{i, j+1}+U_{i-1, j+1}-U_{i+1, j}+2 U_{i, j}-U_{i-1, j}\right)=0
\end{aligned}
$$


for $i=1(1) N-1$ and $j=0(1) J$ with a truncation error of $O(\Delta t)+O(\Delta x)^{2}$. The scheme is a nonlinear system of equations in $U_{i, j+1}$ and it needs to use an iteration technique to evaluate the solution.

Using the central difference operator $\delta$ defined by $\delta_{x} U_{i, j}=U_{i+1, j}-U_{i-1, j}$, (2.5) can be written as

$$
\begin{aligned}
& \frac{U_{i, j+1}-U_{i, j}}{\Delta t}+\frac{1}{4 \Delta x}\left(U_{i+1, j+1}-U_{i-1, j+1}+U_{i+1, j}-U_{i-1, j}\right) \\
& \quad+\frac{\varepsilon}{8 \Delta x}\left\{\delta_{x}\left(U_{i, j+1}^{2}\right)+\delta_{x}\left(U_{i, j}^{2}\right)\right\} \\
& \quad-\frac{\mu}{\Delta t(\Delta x)^{2}}\left(U_{i+1, j+1}-2 U_{i, j+1}+U_{i-1, j+1}-U_{i+1, j}+2 U_{i, j}-U_{i-1, j}\right)=0 .
\end{aligned}
$$

By Taylor expansion of $U_{i, j+1}^{2}$ about the point $(i, j)$ we obtain

$$
U_{i, j+1}^{2}=U_{i, j}^{2}+\Delta t \frac{\partial U_{i, j}^{2}}{\partial t}+\cdots=U_{i, j}^{2}+\Delta t \frac{\partial U_{i, j}^{2}}{\partial U_{i, j}} \frac{\partial U_{i, j}}{\partial t}+\cdots
$$

Hence in terms of order $\Delta t, U_{i, j+1}^{2} \cong U_{i, j}^{2}+2 U_{i, j}\left(U_{i, j+1}-U_{i, j}\right)$, and taking

$$
W_{i}=U_{i, j+1}-U_{i, j}
$$

(2.6), with some manipulations, leads to

$$
\begin{gathered}
\left(\frac{\varepsilon}{4 \Delta x} U_{i-1, j}+\frac{\mu}{\Delta t(\Delta x)^{2}}+\frac{1}{4 \Delta x}\right) W_{i-1}-\left(\frac{1}{\Delta t}+\frac{2 \mu}{\Delta t(\Delta x)^{2}}\right) W_{i} \\
+\left(\frac{\mu}{\Delta t(\Delta x)^{2}}-\frac{1}{4 \Delta x} U_{i+1, j}-\frac{1}{4 \Delta x}\right) W_{i+1} \\
=\frac{1}{2 \Delta x}\left(U_{i+1, j}-U_{i-1, j}\right)+\frac{\varepsilon}{4 \Delta x}\left(U_{i+1, j}^{2}-U_{i-1, j}^{2}\right)
\end{gathered}
$$

$(i=1(1) N-1)$ a system of linear equations for $W_{i}$. This approximation is second order in both space and time as regards truncation error. Obviously, the solution at the $(j+$ 1)th time level is obtained from (2.8) as $U_{i, j+1}=U_{i, j}+W_{i}$. Since the stability parameter $\Delta t /(\Delta x)^{2}$ depends not only on the form of the finite difference scheme (2.9) but also generally upon the solution $U(x, t)$ being obtained, the complications and difficulties may arise in the analysis of stability. In order to show how good the numerical solutions are in comparison with the exact ones, we will use the $L_{2}$ and $L_{\infty}$ error norms defined by

$$
\begin{gathered}
L_{2}=\left\|U^{\text {exact }}-U^{\text {num }}\right\|_{2}=\left[\Delta x \sum_{i=1}^{N}\left|U_{i}^{\text {exact }}-U_{i}^{\text {num }}\right|^{2}\right]^{1 / 2}, \\
L_{\infty}=\left\|U^{\text {exact }}-U^{\text {num }}\right\|_{\infty}=\max _{i}\left|U_{i}^{\text {exact }}-U_{i}^{\text {num }}\right| .
\end{gathered}
$$




\section{Numerical examples and results}

All computations were executed on a Pentium 4 PC in the Fortran code using double precision arithmetic. The RLW equation (1.1) satisfies only three conservation laws given as

$$
\begin{gathered}
I_{1}=\int_{-\infty}^{+\infty} U d x \simeq \Delta x \sum_{i=1}^{N} U_{i, j}, \\
I_{2}=\int_{-\infty}^{+\infty}\left[U^{2}+\mu\left(U_{x}\right)^{2}\right] d x \simeq \Delta x \sum_{i=1}^{N}\left[\left(U_{i, j}\right)^{2}+\mu\left(\left(U_{x}\right)_{i, j}\right)^{2}\right], \\
I_{3}=\int_{-\infty}^{+\infty}\left[U^{3}+3 U^{2}\right] d x \simeq \Delta x \sum_{i=1}^{N}\left[\left(U_{i, j}\right)^{3}+3\left(U_{i, j}\right)^{2}\right]
\end{gathered}
$$

which respectively correspond to mass, momentum, and energy [24]. In the simulations the invariants $I_{1}, I_{2}$, and $I_{3}$ are monitored to check the conservation of the numerical scheme. For the computation of $U_{x}$ in (3.1), we used a central finite difference approximation.

To implement the performance of the method, three test problems will be considered: the motion of a single solitary wave, the interaction of two positive solitary waves, and the undular bore.

3.1. The motion of a single solitary wave. We first consider (1.1) with the boundary conditions $U \rightarrow 0$ as $x \rightarrow \pm \infty$ and the initial condition

$$
U(x, 0)=3 c \operatorname{sech}^{2}\left(k\left(x-x_{0}\right)\right) .
$$

The exact solution of this problem is

$$
U(x, t)=3 c \operatorname{sech}^{2}\left(k\left(x-v t-x_{0}\right)\right) .
$$

This solution corresponds to the motion of a single solitary wave with amplitude $3 c$ and width $k$, initially centered at $x_{0}$, where $v=1+\varepsilon c$ is the wave velocity and $k=(1 /$ $2)(\varepsilon c / \mu v)^{1 / 2}$. This solution will also be used over an interval $a \leq x \leq b$. For this problem the theoretical values of the invariants are [14]

$$
I_{1}=\frac{6 c}{k}, \quad I_{2}=\frac{12 c^{2}}{k}+\frac{48 k c^{2} \mu}{5}, \quad I_{3}=\frac{36 c^{2}}{k}+\frac{144 c^{3}}{5 k}
$$

which are recorded throughout the simulations. For the purpose of comparing with the earlier work, all computations are done for the parameters $\varepsilon=1, \mu=1$, and $x_{0}=0$.

Table 3.1 displays a comparison of the values of the invariants and error norms obtained by the present method with those obtained using the cubic finite difference method developed by Jain et al. [6] and implemented by Gardner et al. [10] for $c=0.1$. As it is seen from the table, the numerical values of invariants obtained from (3.1) are in very good agreement with their analytical values obtained from (3.4). The quantities in the invariants remain almost constant during the computer run. For the proposed finite difference 
Table 3.1. Invariants and error norms for the single soliton with $c=0.1, \Delta x=0.1, \Delta t=0.1$, and over the region $-40 \leq x \leq 60$.

\begin{tabular}{cccccc}
\hline$t$ & $I_{1}$ & $I_{2}$ & $I_{3}$ & $L_{2} \times 10^{3}$ & $L_{\infty} \times 10^{3}$ \\
\hline \multicolumn{5}{c}{ Present method } \\
\hline 0 & 3.97992 & 0.810459 & 2.57901 & 0.00 & 0.00 \\
4 & 3.97995 & 0.810459 & 2.57901 & 0.12 & 0.05 \\
8 & 3.97997 & 0.810459 & 2.57901 & 0.23 & 0.09 \\
12 & 3.97999 & 0.810459 & 2.57901 & 0.34 & 0.14 \\
16 & 3.97999 & 0.810459 & 2.57901 & 0.45 & 0.18 \\
20 & 3.97997 & 0.810459 & 2.57901 & 0.55 & 0.21 \\
\hline \multicolumn{5}{c}{ Finite difference cubic method [6,10] } \\
\hline 0 & 3.97992 & 0.810459 & 2.57901 & 0.00 & 0.00 \\
4 & 4.42017 & 0.899873 & 2.86339 & 39.82 & 13.74 \\
8 & 4.41822 & 0.899236 & 2.86106 & 79.46 & 27.66 \\
12 & 4.41623 & 0.898601 & 2.85863 & 118.8 & 41.35 \\
16 & 4.41423 & 0.897967 & 2.85613 & 157.7 & 54.60 \\
20 & 4.41219 & 0.897342 & 2.85361 & 196.1 & 67.35 \\
\hline
\end{tabular}

method at times $t=0$ and $t=20$, change in $I_{1}$ is $0.5 \times 10^{-4}$, and $I_{2}$ and $I_{3}$ are exact up to the last recorded digit, whereas for the cubic finite difference method, they are 0.43227 , 0.086883 , and 0.2746 , respectively. The error norms at each time obtained by the present method are smaller than those given in $[6,10]$. For the present method at $t=20$, the error norms are $L_{2}=0.55 \times 10^{-3}$ and $L_{\infty}=0.21 \times 10^{-3}$, whereas they are $L_{2}=196.1 \times 10^{-3}$ and $L_{\infty}=67.35 \times 10^{-3}$ for the cubic finite difference method. In Table 3.2 the time evolution of the invariants $I_{1}, I_{2}$, and $I_{3}$, and of the error norms $L_{2}$ and $L_{\infty}$ for $c=0.03$, is compared with the cubic finite difference method $[6,10]$. Again the present method produces good results.

The rates of convergence for the proposed numerical method in space sizes $\Delta x_{m}$ and time steps $\Delta t_{m}$ can be calculated by

$$
\begin{aligned}
& \text { Order }=\frac{\log _{10}\left(\left|U^{\text {exact }}-U_{\Delta x_{m}}^{\text {num }}\right| /\left|U^{\text {exact }}-U_{\Delta x_{m+1}}^{\text {num }}\right|\right)}{\log _{10}\left(\Delta x_{m} / \Delta x_{m+1}\right)}, \\
& \text { Order }=\frac{\log _{10}\left(\left|U^{\text {exact }}-U_{\Delta t_{m}}^{\text {num }}\right| /\left|U^{\text {exact }}-U_{\Delta t_{m+1}}^{\text {num }}\right|\right)}{\log _{10}\left(\Delta t_{m} / \Delta t_{m+1}\right)},
\end{aligned}
$$

respectively [18].

The convergence rates computed by the present method for values of space size $\Delta x_{m}$ and a fixed value of the time step $\Delta t$ are recorded in Table 3.3. It is clearly seen that the scheme provides remarkable reductions in convergence rates for the smaller space sizes. 


\section{Regularized long-wave equation}

Table 3.2. Invariants and error norms for the single soliton with $c=0.03, \Delta x=0.1, \Delta t=0.1$, and over the region $-40 \leq x \leq 60$.

\begin{tabular}{cccccc}
\hline$t$ & $I_{1}$ & $I_{2}$ & $I_{3}$ & $L_{2} \times 10^{3}$ & $L_{\infty} \times 10^{3}$ \\
\hline \multicolumn{5}{c}{ Present method } \\
\hline 0 & 2.107 & 0.127301 & 0.388804 & 0.000 & 0.000 \\
4 & 2.108 & 0.127302 & 0.388806 & 0.150 & 0.123 \\
8 & 2.109 & 0.127302 & 0.388807 & 0.321 & 0.166 \\
12 & 2.110 & 0.127302 & 0.388807 & 0.467 & 0.179 \\
16 & 2.110 & 0.127302 & 0.388808 & 0.567 & 0.185 \\
20 & 2.109 & 0.127302 & 0.388807 & 0.638 & 0.233 \\
\hline \multicolumn{5}{c}{ Finite difference cubic method [6,10] } \\
\hline 0 & 2.107 & 0.127301 & 0.388804 & 0.000 & 0.000 \\
4 & 2.340 & 0.141322 & 0.431621 & 2.928 & 0.786 \\
8 & 2.339 & 0.141195 & 0.431231 & 5.816 & 1.582 \\
12 & 2.337 & 0.141067 & 0.430834 & 8.698 & 2.384 \\
16 & 2.336 & 0.140940 & 0.430440 & 11.58 & 3.190 \\
20 & 2.333 & 0.140815 & 0.430052 & 14.45 & 3.996 \\
\hline
\end{tabular}

Table 3.3. The order of convergence at $t=20, \Delta t=0.1, c=0.1(-40 \leq x \leq 60)$, and $c=0.03(-80 \leq$ $x \leq 120)$.

\begin{tabular}{cccccc}
\hline$c$ & $\Delta x_{j}$ & $L_{2} \times 10^{3}$ & Order & $L_{\infty} \times 10^{3}$ & Order \\
\hline 0.1 & 1 & 33.66668 & - & 12.74833 & - \\
& 0.5 & 8.767886 & 1.941021 & 3.381133 & 1.914730 \\
& 0.25 & 2.358203 & 1.894541 & 0.910513 & 1.892755 \\
& 0.125 & 0.744691 & 1.662974 & 0.286720 & 1.667037 \\
& 0.025 & 0.229367 & 0.731713 & 0.086429 & 0.745094 \\
& 0.0125 & 0.213601 & 0.102739 & 0.080163 & 0.108579 \\
\hline 0.03 & 1 & 2.620662 & - & 0.794513 & - \\
& 0.5 & 0.667923 & 1.972178 & 0.202298 & 1.973589 \\
& 0.25 & 0.177379 & 1.912847 & 0.053656 & 1.914671 \\
& 0.125 & 0.054606 & 1.699704 & 0.016471 & 1.703811 \\
& 0.025 & 0.015359 & 0.788127 & 0.004569 & 0.796742 \\
& 0.0125 & 0.014146 & 0.118690 & 0.004198 & 0.122176 \\
\hline
\end{tabular}

Table 3.4 displays the computed convergence rates for various values of time step $\Delta t_{j}$ and a fixed value of the space size $\Delta x$. Again a noticeable decrease in convergence rates is observed when the time step decreases. 
Table 3.4. The order of convergence at $t=20, \Delta x=0.1, c=0.1(-40 \leq x \leq 60)$, and $c=0.03(-80 \leq$ $x \leq 120)$.

\begin{tabular}{cccccc}
\hline$c$ & $\Delta t_{j}$ & $L_{2} \times 10^{3}$ & Order & $L_{\infty} \times 10^{3}$ & Order \\
\hline 0.1 & 1 & 20.292460 & - & 7.618319 & - \\
& 0.5 & 5.461549 & 1.893562 & 2.062621 & 1.884994 \\
& 0.25 & 1.631432 & 1.743171 & 6.197000 & 1.734837 \\
& 0.125 & 0.666724 & 1.290977 & 0.255439 & 1.278591 \\
& 0.025 & 0.358400 & 0.385679 & 0.138568 & 0.380022 \\
& 0.0125 & 0.348799 & 0.039175 & 0.134914 & 0.038554 \\
\hline 0.03 & 1 & 1.380075 & - & 0.411315 & - \\
& 0.5 & 0.367151 & 1.910301 & 0.109469 & 1.909721 \\
& 0.25 & 0.111587 & 1.718205 & 0.033363 & 1.714201 \\
& 0.125 & 0.047558 & 1.230409 & 0.014296 & 1.222637 \\
& 0.025 & 0.027068 & 0.350183 & 0.008194 & 0.345821 \\
& 0.0125 & 0.026428 & 0.034521 & 0.008003 & 0.034027 \\
\hline
\end{tabular}

The profiles of the solitary waves at times $t=0$ and $t=20$ and the error distributions of the analytical and numerical solutions at $t=20$ for $c=0.1$ with the range $-40 \leq x \leq 60$ and for $c=0.03$ with the range $-80 \leq x \leq 120, \Delta x=0.125$ and $\Delta t=0.1$ are shown in Figure 3.1. For $c=0.1$, the amplitude is 0.3 at time $t=0$ while it is 0.299919 at time $t=20$ (Figure 3.1(a)) and so the relative change in the amplitude is about $0.027 \%$. It is seen that the maximum error is about between $-4 \times 10^{-3}$ and $4 \times 10^{-3}$ (Figure 3.1(b)). For $c=$ 0.03 , the amplitude is 0.09 at time $t=0$ while it is 0.089997 at time $t=20$ (Figure 3.1(c)) and so the relative change in the amplitude is about $0.0033 \%$. It is observed that the maximum error is about between $-6 \times 10^{-4}$ and $6 \times 10^{-4}$ (Figure 3.1(d)).

3.2. The interaction of two positive solitary waves. We secondly consider (1.1) with the boundary conditions $U \rightarrow 0$ as $x \rightarrow \pm \infty$ and the initial condition [17]

$$
U(x, 0)=\sum_{j=1}^{2} 3 A_{j} \operatorname{sech}^{2}\left(k_{j}\left(x-x_{j}\right)\right)
$$

where $A_{j}=4 k_{j}^{2} /\left(1-4 k_{j}^{2}\right)(j=1,2)$.

For the simulation, all computations are done for the parameters $k_{1}=0.4, x_{1}=15$, $k_{2}=0.3, x_{2}=35, \varepsilon=1, \mu=1, \Delta x=0.3$, and $\Delta t=0.1$ over the region $0 \leq x \leq 120$. The experiment was run from $t=0$ to $t=25$ to allow the interaction to take place. Figure 3.2 shows the interaction of two positive solitary waves. As it is seen from the figure, at $t=0$ a solitary wave with larger amplitude is on the left of the other solitary wave with smaller amplitude. The larger wave catches up with the smaller one as the time increases. At $t=0$, 


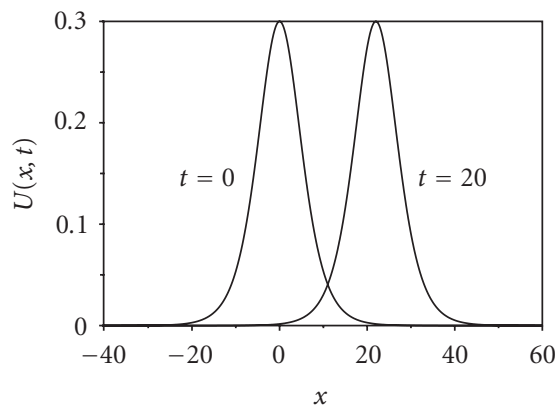

(a)

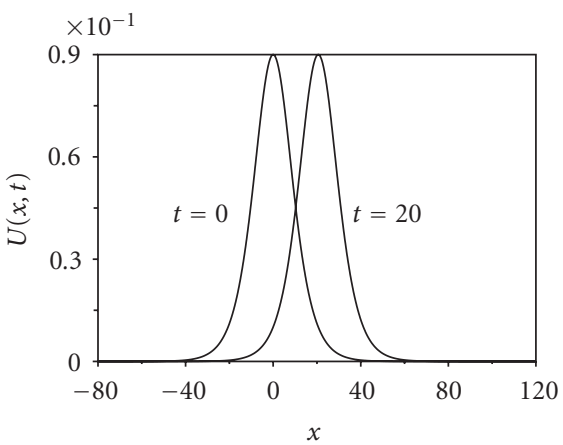

(c)

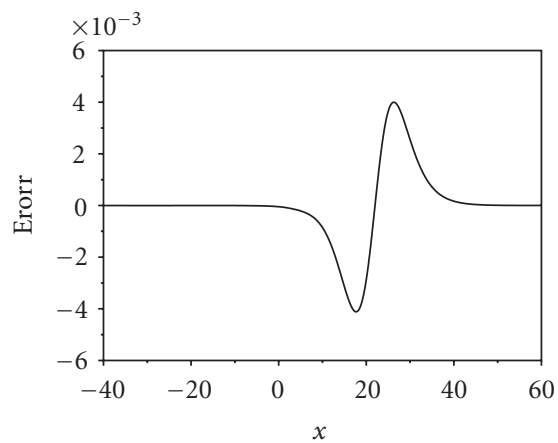

(b)

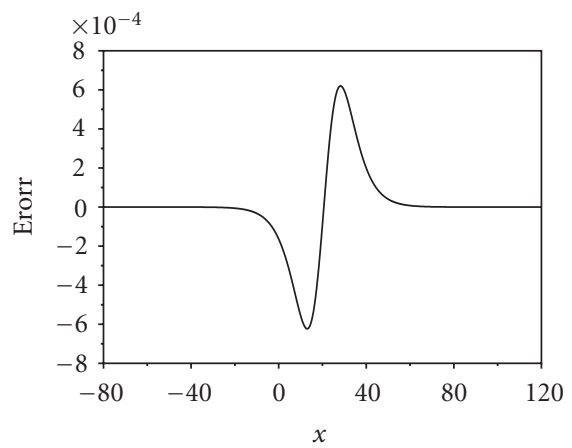

(d)

Figure 3.1. Solitary wave profiles at $t=0,20$ and error (error $=$ exact-numerical) distributions at $t=20$.

the amplitude of the larger solitary wave is 5.33338 while the amplitude of the smaller one is 1.68598 , whereas at $t=25$, the amplitude of the larger solitary wave is 5.30235 at the point $x=86.7$ while the amplitude of the smaller one is 1.67157 at the point $x=69.9$. An oscillation of small amplitude trailing behind the solitary waves was observed. In order to see this oscillation occurring behind the waves in Figure 3.2 at time $t=25$, the scale of the figure is magnified as in Figure 3.3. It is clearly seen that an oscillation of amplitude $\sim 2.2 \times 10^{-2}$ is trailing behind the solitary waves.

Table 3.5 displays a comparison of the values of the invariants obtained by the present method with those obtained in [17]. It is observed that the obtained values of the invariants remain almost constant during the computer run. At times $t=0$ and $t=25$, the relative changes in the invariants $I_{1}, I_{2}$, and $I_{3}$ for the present method are respectively $2.558 \times 10^{-3} \%, 6.647 \times 10^{-3} \%$, and $9.797 \times 10^{-3} \%$ whereas they are $0.352 \%, 0.570 \%$, and $2.237 \%$ for the cubic B-spline collocation finite element method given in [17]. It is clearly seen that each of the conserved quantities obtained by the present method is very well preserved. 


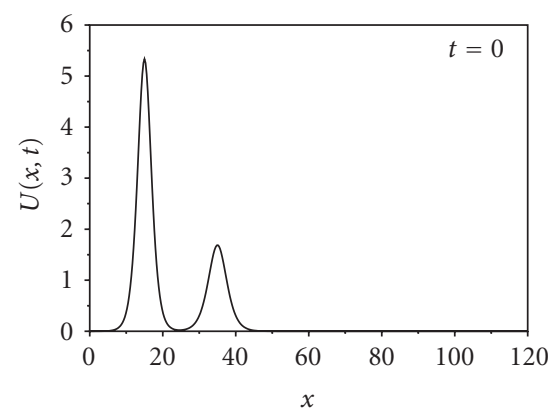

(a)

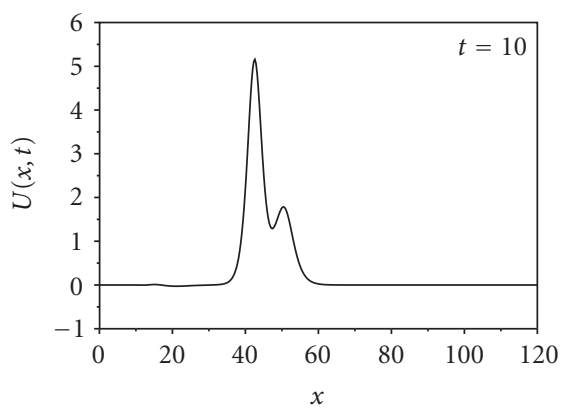

(c)

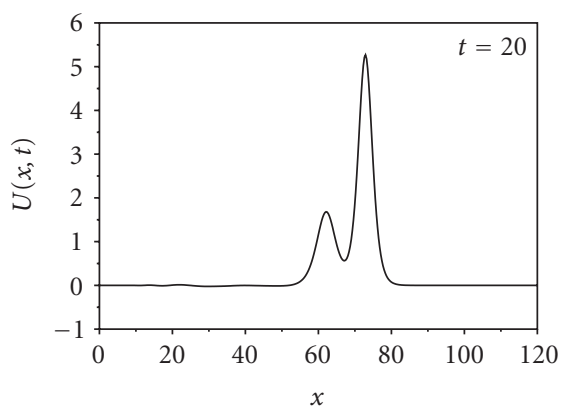

(e)

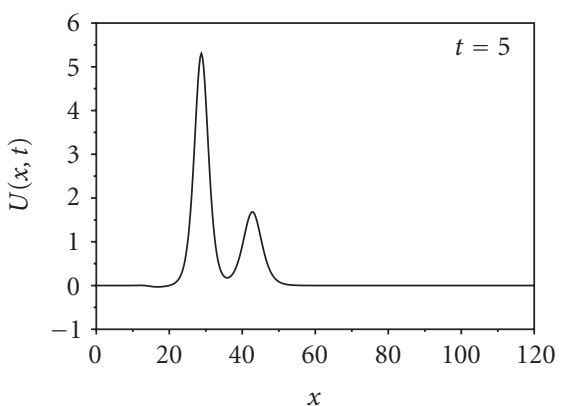

(b)

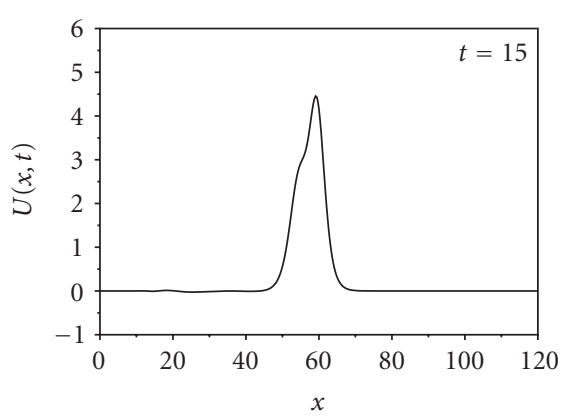

(d)

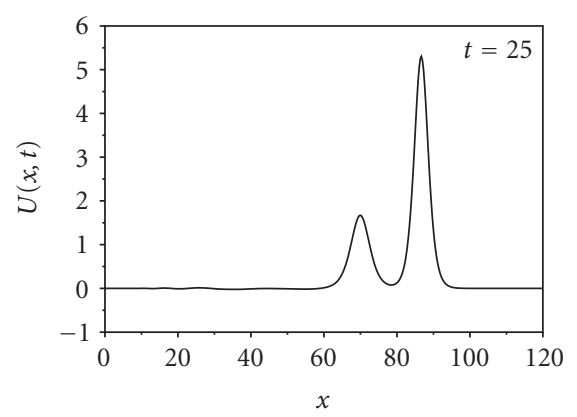

(f)

Figure 3.2. The interaction of two positive solitary waves at different times.

3.3. The undular bore. As our last test problem, we consider (1.1) with the physical boundary conditions $U \rightarrow 0$ as $x \rightarrow \infty$ and $U \rightarrow U_{0}$ as $x \rightarrow-\infty$, and the initial condition

$$
U(x, 0)=\frac{U_{0}}{2}\left[1-\tanh \left(\frac{x-x_{0}}{d}\right)\right]
$$




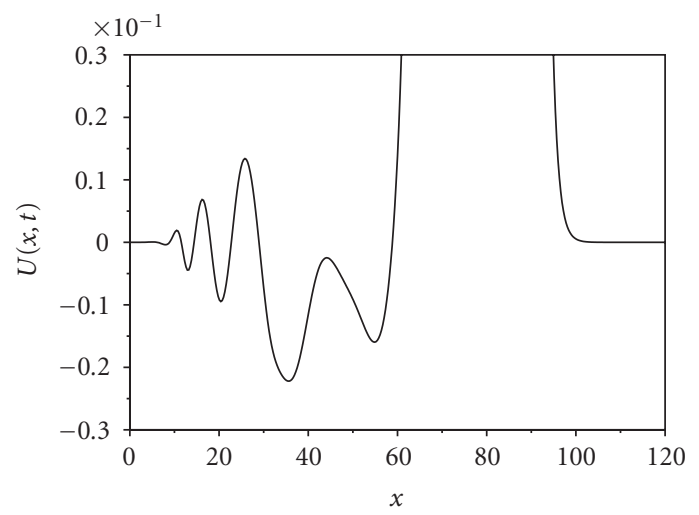

Figure 3.3. The interaction of two solitary waves at $t=25$ in Figure 3.2 (magnified).

where $U(x, 0)$, denotes the elevation of the water surface above the equilibrium level at time $t=0, U_{0}$ represents the magnitude of the change in water level which is centered on $x=x_{0}$, and $d$ measures the steepness of the change. Under the above physical boundary conditions, the invariants $I_{1}, I_{2}, I_{3}$ are not constant but increase linearly throughout the simulation at the following rates [14]:

$$
\begin{gathered}
M_{1}=\frac{d}{d t} I_{1}=\frac{d}{d t} \int_{-\infty}^{+\infty} U d x=U_{0}+\frac{1}{2} U_{0}^{2}, \\
M_{2}=\frac{d}{d t} I_{2}=\frac{d}{d t} \int_{-\infty}^{+\infty}\left\{U^{2}+\mu\left(U_{x}\right)^{2}\right\} d x=U_{0}^{2}+\frac{2}{3} U_{0}^{3}, \\
M_{3}=\frac{d}{d t} I_{3}=\frac{d}{d t} \int_{-\infty}^{+\infty}\left(U^{3}+3 U^{2}\right) d x=3 U_{0}^{2}+3 U_{0}^{3}+\frac{3}{4} U_{0}^{4},
\end{gathered}
$$

respectively.

For the simulation, all computations are done for the parameters $\varepsilon=1.5, \mu=1 / 6$, $U_{0}=0.1, x_{0}=0, \Delta x=0.24, \Delta t=0.1$, and $d=2,5$ in the region $-36 \leq x \leq 300$. The simulation is run until time $t=250$, and the values of the quantities $I_{1}, I_{2}, I_{3}$ with the position and amplitude of the leading undulation for the steep slope $d=2$ and the gentle slope $d=5$ are recorded in Table 3.6. The numerical values of variations in quantities $I_{1}, I_{2}, I_{3}$ are obtained as $M_{1}=0.107500, M_{2}=0.010992, M_{3}=0.034096$ for $d=2$ and $M_{1}=0.107500, M_{2}=0.010992, M_{3}=0.034101$ for $d=5$ which are in good agreement with the theoretical values $M_{1}=0.105000, M_{2}=0.010667, M_{3}=0.033075$ obtained from (3.8). The values of $I_{1}, I_{2}$, and $I_{3}$ increase linearly according to the values of $M_{1}, M_{2}$, and $M_{3}$, respectively. The amplitudes of the leading undulation for $d=5$ and $d=2$ are 0.17710 and 0.18158 , respectively. 
Table 3.5. Invariants for the interaction of two positive solitary waves.

\begin{tabular}{ccccccc}
\hline$t$ & $I_{1}$ & $I_{2}$ & $I_{3}$ & $I_{1}[17]$ & $I_{2}[17]$ & $I_{3}[17]$ \\
\hline 0 & 37.91648 & 120.35150 & 744.08140 & 37.91652 & 120.52280 & 744.08150 \\
2 & 37.91682 & 120.35710 & 744.03870 & 37.91596 & 119.17830 & 725.54580 \\
4 & 37.91697 & 120.35840 & 744.01100 & 37.91170 & 121.16020 & 736.94430 \\
5 & 37.91704 & 120.35860 & 743.99850 & - & - & - \\
6 & 37.91709 & 120.35830 & 743.97960 & 37.89662 & 118.12660 & 714.05840 \\
8 & 37.91719 & 120.35700 & 743.86790 & 37.85975 & 119.73170 & 728.51730 \\
10 & 37.91727 & 120.36380 & 743.42020 & 37.79221 & 119.73430 & 726.68790 \\
12 & 37.91733 & 120.39150 & 742.33870 & 37.69667 & 119.63340 & 725.72360 \\
14 & 37.91736 & 120.41560 & 741.57810 & 37.59553 & 119.23590 & 724.70020 \\
15 & 37.91738 & 120.40600 & 741.89150 & - & - & - \\
16 & 37.91740 & 120.38860 & 742.48890 & 37.52916 & 119.41850 & 725.83990 \\
18 & 37.91741 & 120.36530 & 743.47520 & 37.54027 & 119.82760 & 727.08860 \\
20 & 37.91744 & 120.35990 & 743.86380 & 37.64730 & 119.80410 & 727.19480 \\
22 & 37.91745 & 120.35940 & 743.97500 & 37.82237 & 119.79820 & 727.25420 \\
24 & 37.91746 & 120.35950 & 744.00370 & 37.99313 & 119.89230 & 727.49210 \\
25 & 37.91745 & 120.35950 & 744.00850 & 38.05010 & 119.83550 & 727.43920 \\
\hline & & & & & & \\
\hline
\end{tabular}

Table 3.6. Invariants, position, and amplitude of the leading undulation for $d=2,5$.

\begin{tabular}{|c|c|c|c|c|c|c|}
\hline$d$ & $t$ & $I_{1}$ & $I_{2}$ & $I_{3}$ & $x$ & Amplitude \\
\hline \multirow[t]{6}{*}{2} & 0 & 3.58800 & 0.35081 & 1.08078 & - & - \\
\hline & 50 & 8.96300 & 0.89905 & 2.78584 & 48.96000 & 0.13940 \\
\hline & 100 & 14.33799 & 1.44901 & 4.49069 & 102.48000 & 0.15831 \\
\hline & 150 & 19.71301 & 1.99896 & 6.19543 & 156.72000 & 0.17013 \\
\hline & 200 & 25.08799 & 2.54892 & 7.90013 & 211.20000 & 0.17713 \\
\hline & 250 & 30.46299 & 3.09887 & 9.60482 & 265.68000 & 0.18158 \\
\hline \multirow[t]{6}{*}{5} & 0 & 3.58800 & 0.33565 & 1.03353 & - & - \\
\hline & 50 & 8.96300 & 0.88391 & 2.73902 & 48.24000 & 0.11067 \\
\hline & 100 & 14.33801 & 1.43389 & 4.44424 & 102.24000 & 0.13683 \\
\hline & 150 & 19.71300 & 1.98385 & 6.14918 & 156.24000 & 0.15714 \\
\hline & 200 & 25.08802 & 2.53381 & 7.85395 & 210.48000 & 0.16990 \\
\hline & 250 & 30.46305 & 3.08376 & 9.55868 & 264.96000 & 0.17710 \\
\hline
\end{tabular}




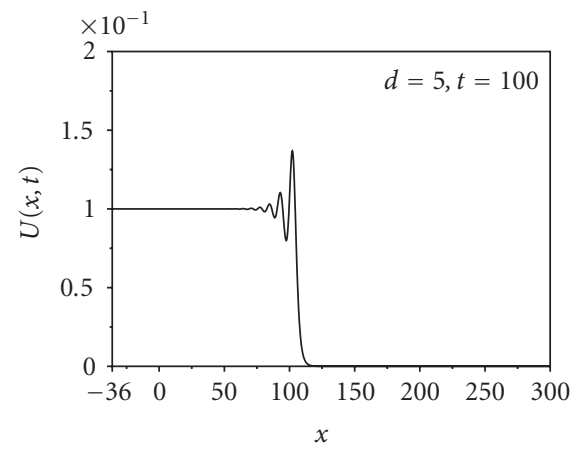

(a)

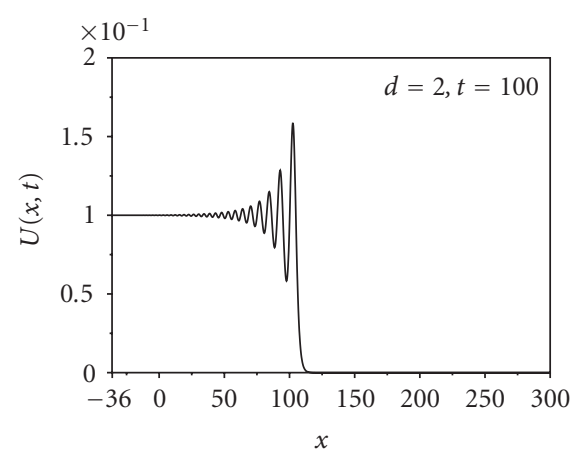

(c)

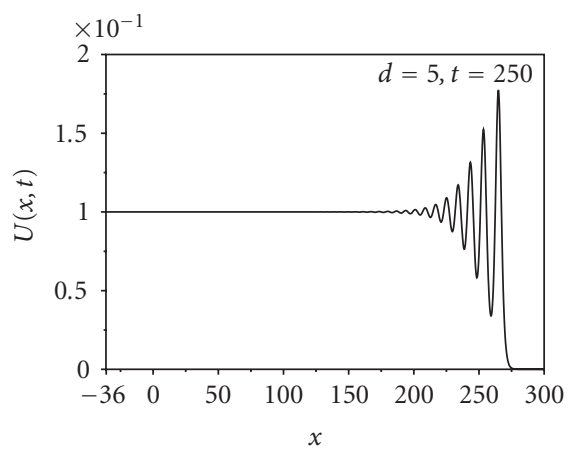

(b)

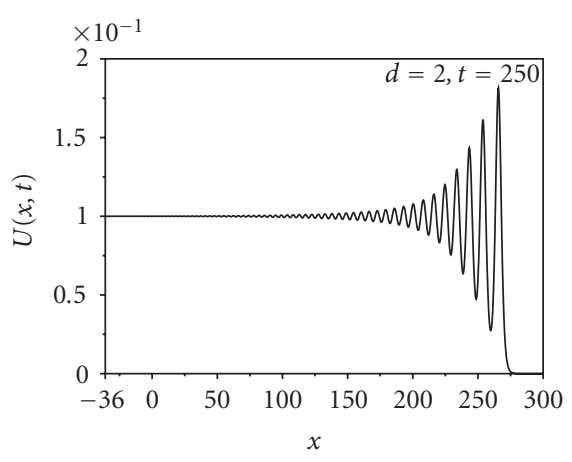

(d)

Figure 3.4. Undulation profiles for the gentle slope $d=5$ and steep slope $d=2$ at $t=100$ and $t=250$.

Figure 3.4 illustrates the undular bore profiles at $t=100$ and $t=250$ for the gentle slope $d=5$ and the steep slope $d=2$. As it can be seen that from the figure, the number of undulations formed increases with the decrease of $d$ from $d=5$ to $d=2$. The number of undulations also increases with the increase of $t$, as expected.

\section{Conclusion}

A linearized implicit finite difference method was presented to obtain numerical solutions of the RLW equation. The efficiency of the method was tested on three numerical experiments of wave propagation: the motion of a single solitary wave, the development of two positive solitary waves interaction, and an undular bore, and its accuracy was examined by the error norms $L_{2}$ and $L_{\infty}$. The obtained results show that the error norms are reasonably small and the conservation properties are all very good. The results also suggest that the present method whose application is easier than many other numerical techniques such as finite element and spectral methods can be applied to a large number of physically important nonlinear wave problems with success. 


\section{References}

[1] D. H. Peregrine, Calculations of the development of an undular bore, Journal of Fluid Mechanics 25 (1966), 321-330.

[2] T. B. Benjamin, J. L. Bona, and J. J. Mahony, Model equations for long waves in nonlinear dispersive systems, Philosophical Transactions of the Royal Society of London. Series A 272 (1972), no. $1220,47-78$.

[3] J. L. Bona and P. J. Bryant, A mathematical model for long waves generated by wavemakers in non-linear dispersive systems, Proceedings of the Cambridge Philosophical Society 73 (1973), 391-405.

[4] P. C. Jain and L. Iskandar, Numerical solutions of the regularized long-wave equation, Computer Methods in Applied Mechanics and Engineering 20 (1979), no. 2, 195-201.

[5] J. C. Eilbeck and G. R. McGuire, Numerical study of the regularized long-wave equation. II. Interaction of solitary waves, Journal of Computational Physics 23 (1977), no. 1, 63-73.

[6] P. C. Jain, R. Shankar, and T. V. Singh, Numerical solution of regularized long-wave equation, Communications in Numerical Methods in Engineering 9 (1993), no. 7, 579-586.

[7] D. Bhardwaj and R. Shankar, A computational method for regularized long wave equation, Computers \& Mathematics with Applications 40 (2000), no. 12, 1397-1404.

[8] Q. S. Chang, G. B. Wang, and B. L. Guo, Conservative scheme for a model of nonlinear dispersive waves and its solitary waves induced by boundary motion, Journal of Computational Physics 93 (1991), no. 2, 360-375.

[9] L. R. T. Gardner and G. A. Gardner, Solitary waves of the regularised long-wave equation, Journal of Computational Physics 91 (1990), no. 2, 441-459.

[10] L. R. T. Gardner, G. A. Gardner, and I. Dag, A B-spline finite element method for the regularized long wave equation, Communications in Numerical Methods in Engineering 11 (1995), no. 1, $59-68$.

[11] I. Dag, Least-squares quadratic B-spline finite element method for the regularised long wave equation, Computer Methods in Applied Mechanics and Engineering 182 (2000), no. 1-2, 205-215.

[12] I. Dag and M. N. Özer, Approximation of RLW equation by least square cubic B-spline finite element method, Applied Mathematical Modelling 25 (2001), no. 3, 221-231.

[13] A. Dogan, Numerical solution of RLW equation using linear finite elements within Galerkin's method, Applied Mathematical Modelling 26 (2002), no. 7, 771-783.

[14] S. I. Zaki, Solitary waves of the splitted RLW equation, Computer Physics Communications 138 (2001), no. 1, 80-91.

[15] I. Dag, B. Saka, and D. Irk, Application of cubic B-splines for numerical solution of the RLW equation, Applied Mathematics and Computation 159 (2004), no. 2, 373-389.

[16] A. A. Soliman and K. R. Raslan, Collocation method using quadratic B-spline for the RLW equation, International Journal of Computer Mathematics 78 (2001), no. 3, 399-412.

[17] K. R. Raslan, A computational method for the regularized long wave (RLW) equation, Applied Mathematics and Computation 167 (2005), no. 2, 1101-1118.

[18] I. Dag, B. Saka, and D. Irk, Galerkin method for the numerical solution of the RLW equation using quintic B-splines, Journal of Computational and Applied Mathematics 190 (2006), no. 1-2, 532547.

[19] B. Y. Guo and W. M. Cao, The Fourier pseudospectral method with a restrain operator for the RLW equation, Journal of Computational Physics 74 (1988), no. 1, 110-126.

[20] B. Y. Guo and V. S. Manoranjan, Spectral method for solving the RLW equation, Journal of Computational Mathematics 3 (1985), no. 3, 228-237.

[21] D. M. Sloan, Fourier pseudospectral solution of the regularised long wave equation, Journal of Computational and Applied Mathematics 36 (1991), no. 2, 159-179. 


\section{Regularized long-wave equation}

[22] K. Djidjeli, W. G. Price, E. H. Twizell, and Q. Cao, A linearized implicit pseudo-spectral method for some model equations: the regularized long wave equations, Communications in Numerical Methods in Engineering 19 (2003), no. 11, 847-863.

[23] P. M. Prenter, Splines and Variational Methods, Wiley-Interscience, New York, 1975.

[24] P. J. Olver, Euler operators and conservation laws of the BBM equation, Mathematical Proceedings of the Cambridge Philosophical Society 85 (1979), no. 1, 143-160.

S. Kutluay: Department of Mathematics, Faculty of Arts and Science, Inonu University, 44280 Malatya, Turkey

E-mail address: skutluay@inonu.edu.tr

A. Esen: Department of Mathematics, Faculty of Arts and Science, Inonu University, 44280 Malatya, Turkey

E-mail address: aesen@inonu.edu.tr 


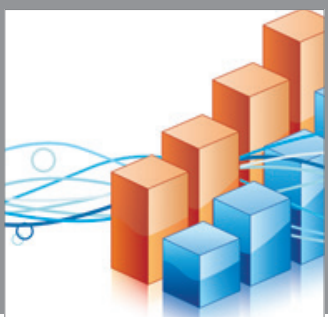

Advances in

Operations Research

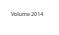

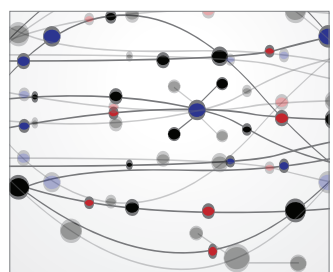

\section{The Scientific} World Journal
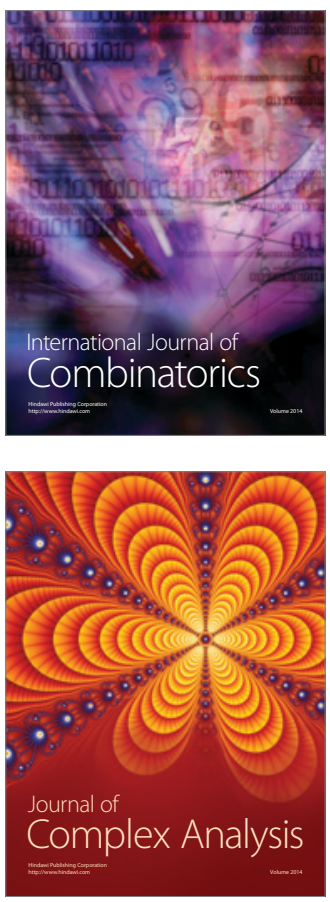

International Journal of

Mathematics and

Mathematical

Sciences
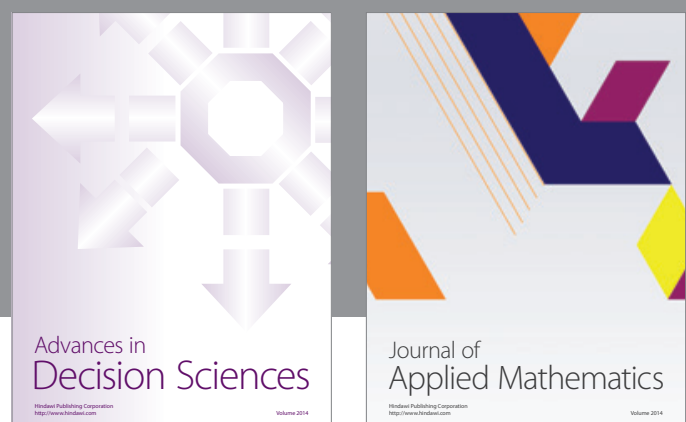

Journal of

Applied Mathematics
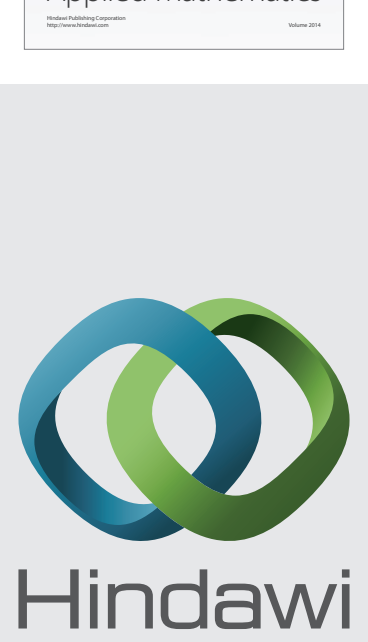

Submit your manuscripts at http://www.hindawi.com
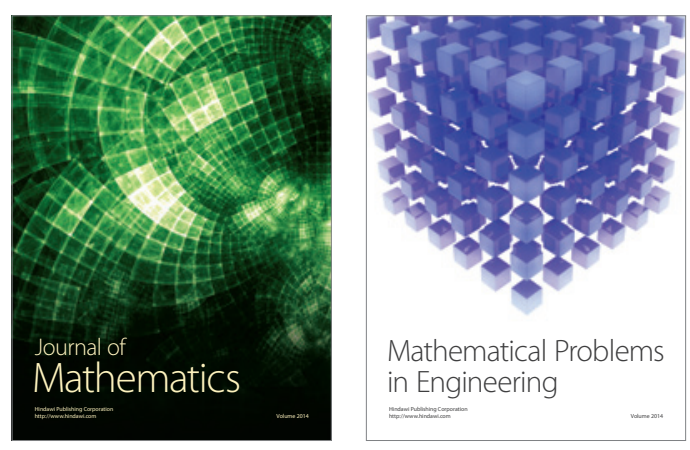

Mathematical Problems in Engineering
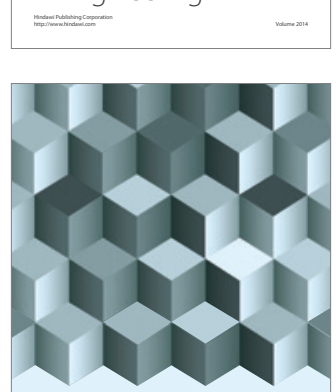

Journal of

Function Spaces
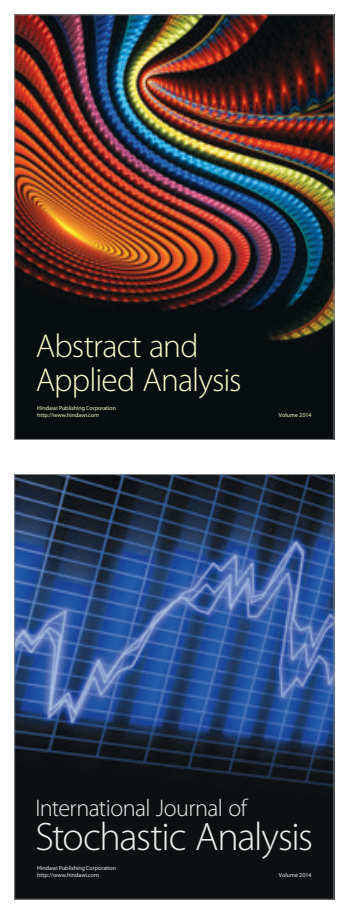

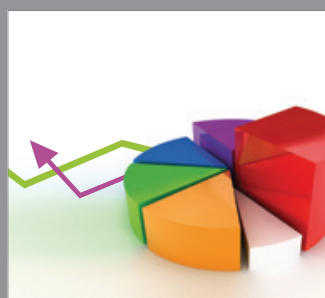

ournal of

Probability and Statistics

Promensencen
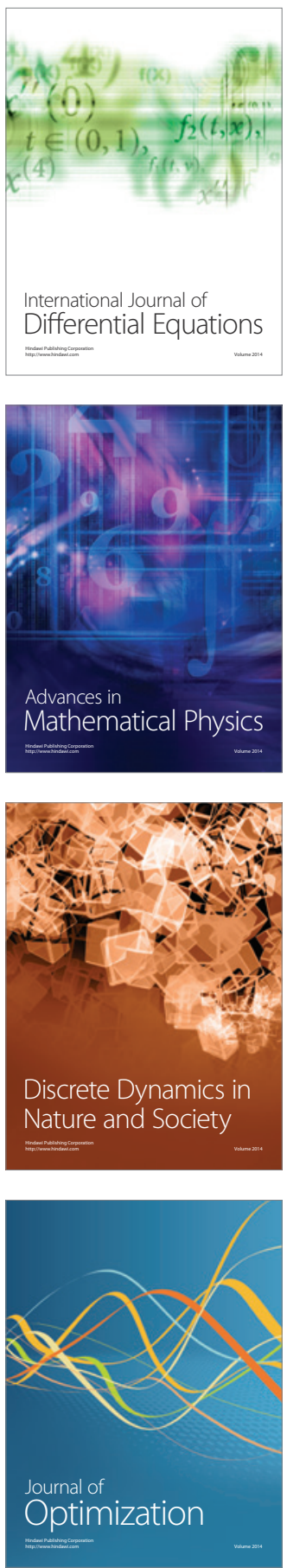